C-A/AP/\#181

December 2004

\title{
Physics of Super Pulses in Storage Ring Free-Electron Lasers
}

\author{
Vladimir N. Litvinenko
}

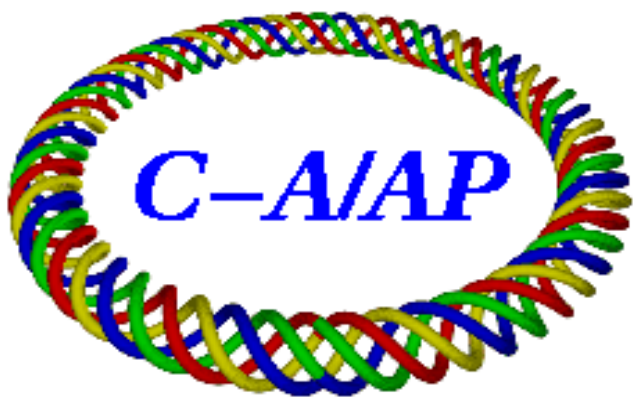

Collider-Accelerator Department Brookhaven National Laboratory

Upton, NY 11973 


\title{
Physics of super pulses in storage ring free-electron lasers
}

\author{
Vladimir N. Litvinenko \\ Collider-Accelerator Department, Brookhaven National Laboratory, Upton, NY 11973
}

\begin{abstract}
Contradictory to the widespread perception, storage ring free-electron lasers with substantial net gain can generate peak lasing power reaching GW levels in the so-called Super-pulse mode. This power level is sufficient for studies of nonlinear processes and efficient intra-cavity harmonic generation. This letter describes the physics of Super-pulses and "phase-space refreshment" of the electron beam responsible for this phenomenon.
\end{abstract}

PACS numbers: 41.60.Cr, 29.20D, 78.66, 52.75

Keywords: Free Electron Laser, Storage Ring, Optical Klystron, Peak Power

All presently existing storage ring free electron lasers (SR FELs) are oscillators with a two-mirror optical cavity and an optical klystron (OK) [1] comprising two wigglers and a buncher between them ${ }^{1}$. SR FELs can operate in pulsed mode [2] with significant peak optical power. Early theoretical models [1] predicted peak intra-cavity (IC) optical power $P_{\text {opt }}$ of:

$$
P_{o p t} \leq P_{e-\text { beam }} \cdot \sigma_{\varepsilon \text { ind }} ; \quad P_{e-\text { beam }}=\frac{\hat{I}_{e} \cdot E_{o}}{e} ;
$$

where $E_{o}=\gamma_{o} m c^{2} ; \gamma_{o}>>1$ is the central energy and $\hat{I}_{e}$ is the peak current of the e-beam, $\sigma_{\varepsilon}$ ind is a relative RMS energy spread induced by FEL interactions and $e$ is the electron charge. For a typical SR FEL with $E_{o} \sim 1 \mathrm{GeV}, \hat{I}_{e} \sim 10 \mathrm{~A}$ and $\sigma_{\text {eind }} \sim 0.1 \%$, eq. (1) limits peak FEL power to about $10 \mathrm{MW}$.

Studies of self-consistent SR FEL dynamics using the 3-D uvfel code [5] revealed a mechanism for generating Superpulses with IC peak power two-to-three orders of magnitude above the estimate given by (1). Fig. 1 shows the typical evolution of a Super-pulse in the OK-4/Duke SR FEL predicted by the uvfel code. Our uvfel code [5] includes most SR FEL effects and has been proven very reliable in predicting both e-beam and optical beam parameters in SR FELs $[2,4,6,7]$.

In this letter we discuss phenomena responsible for Superpulses in SR FEL ${ }^{2}$. We present a self-consistent set of dimensionless non-linear differential equation describing Super-pulse and the peak power dependencies on key parameters. We also discuss the physics of Super-pulses using results of computer simulations for illustration ${ }^{3}$.

An ultra-relativistic electron is described in 6-D phase space by its energy $E_{e}=E_{o}(1+\varepsilon)$, longitudinal coordinate $\xi=v_{e} t-z, \quad$ transverse coordinates and angles $X^{T} \equiv\left[x, x^{\prime}, y, y^{\prime}\right]$, where $x^{\prime} \equiv d x / d z$ and $z$ is the coordinate along the FEL axis. Without lasing, the electron beam obtains

\footnotetext{
${ }^{1}$ For simplicity, we chose round-trip times of the laser pulse in the optical cavity and the electron beam (e-beam) in the ring to be equal. Small deviations from exact synchronism are not important in a pulse SR FEL mode (see $[3,4]$ for details) and the extension to the case of integer ratio between the round trip times is straightforward.

${ }^{2}$ Experiments with Super pulses are published elsewhere [2,7]. The experiments with efficient harmonic generation support our predictions for Super-pulse.

${ }^{3} 10^{5}$ to $10^{6}$ macro-particles were used in simulations. The data used in this letter represent about 1,000 runs of $u v f e l$ code with total number of macro-particle passes through the FEL $\sim 10^{11}$.
}

a natural distribution in 6-D phase space, which is close to a Gaussian and is the product of the normalized transverse $(t)$ and longitudinal $(s)$ distribution functions $f_{6 D} \cong N_{e} \cdot f_{t}(X) \cdot f_{s}$, where $N_{e}$ is the number of electrons in the e-bunch [8]. This separation is valid for processes in SR FELs discussed in this letter ${ }^{4}$ which have time scales much shorter than that of radiation damping in a storage ring.

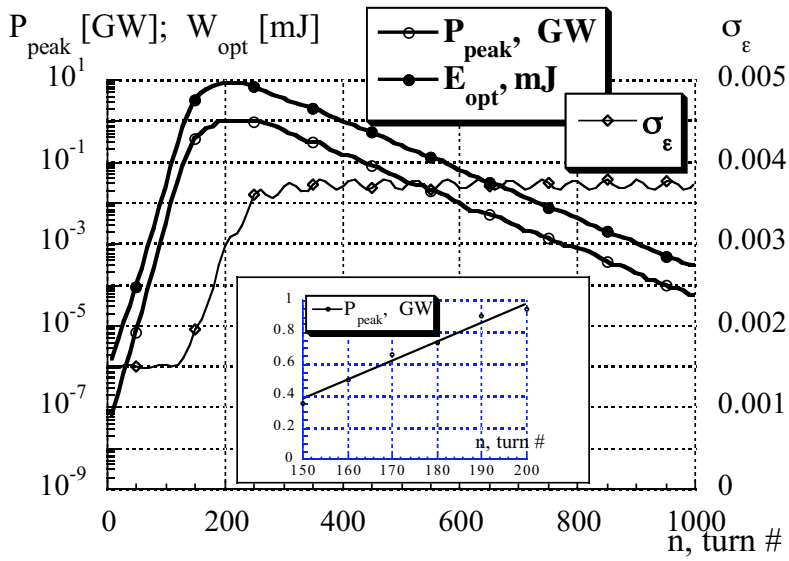

FIG.1. Evolution of a Super-pulse in OK-4/Duke SR FEL predicted by $u v f e l$ code. Intra-cavity optical laser beam energy (๑) and peak power $(\mathrm{O})$ are plotted in log-scale (left), and relative RMS e-beam energy spread $(\diamond)$ is plotted in linear scale (right). Operational parameters: $E_{o}=650 \mathrm{MeV} ; I_{e}=2 \mathrm{~mA}, \hat{I}_{e}=36.3 \mathrm{~A}, \lambda=237 \mathrm{~nm} ; N_{w}=33.5$; $N_{D}=10.75 ; Q_{s}=0.0094, \sigma_{\varepsilon o}=0.15 \%, \sigma_{z o}=2.42 \mathrm{~cm}$; optical cavity losses per turn are $2.01 \%$. Horizontal and vertical emittances are 11.1 and $1.33 \mathrm{~nm}$ rad, $\beta_{x}=3.3 \mathrm{~m} ; \beta_{y}=4 \mathrm{~m}$; the Rayleigh range is $\beta_{o}=2 \mathrm{~m}$ (see [6] for details). Starting peak gain $G_{o}=16.5 \%$. Inset shows peak-power growth (in linear scale) for turns 150-200.

In the absence of FEL interactions, longitudinal motion of the electrons is a small amplitude synchrotron oscillation with frequency $\Omega_{s}=2 \pi Q_{s}$, where $Q_{s}$ is the synchrotron tune [8]:

$$
\delta+i \zeta=a_{s} \cdot e^{i\left(n \Omega_{s}+\varphi_{s}\right)} ; f_{s}(\delta, \zeta) \cong \frac{1}{2 \pi} e^{-\frac{\delta^{2}+\zeta^{2}}{2}} ;
$$

where $n$ is the turn number around the ring, and $\left\{\delta=\varepsilon / \sigma_{\varepsilon o}, \zeta=\xi / \sigma_{z o}\right\}$ are dimensionless coordinates scaled by the natural e-beam energy spread $\sigma_{\varepsilon o}=m c^{2} \sigma_{\gamma o}$ and the bunch length $\sigma_{z o}$. Trajectories in $\{\delta, \zeta\}$ phase-space are simple clock-wise circular rotations (see Fig. 4).

\footnotetext{
${ }^{4}$ i.e. for a SR FEL in the dispersion-free straight section.
} 
The FEL wigglers (with period $\lambda_{w}$ and magnetic field $B_{w}$ ) provide a resonant interaction between electrons and the TEM optical wave at wavelength $\lambda_{o}$ given by:

$$
\lambda_{o}=\frac{\lambda_{w}}{2 \gamma_{o}^{2}}\left(1+\left\langle\vec{a}_{w}^{2}\right\rangle\right) ; \quad \vec{a}_{w}=\frac{e \vec{B}_{w} \lambda_{w}}{2 \pi m c^{2}}
$$

which is a consequence of the electrons slipping behind the optical wave at the rate of:

$$
\frac{d \xi}{d z}=\frac{1+\vec{a}_{w}^{2}(x, y)}{2 \gamma_{o}^{2}}(1-2 \varepsilon)+\frac{x^{\prime 2}+y^{\prime 2}}{2} .
$$

Passing through an $\mathrm{OK}$, an electron radiates a wave-packet with length equal to its total slippage $\Xi \ll \sigma_{z o}$ :

$$
\Xi=\left(2 N_{W}+N_{D}+O\left(\varepsilon, x^{\prime 2}, y^{\prime 2}\right)\right) \lambda_{o},
$$

where $N_{w}$ is the number of periods in one wiggler and $N_{D}$ is the dimensionless slippage in the buncher [9]. When FEL interactions are present, the intra-cavity optical power builds up from spontaneous radiation, which is accumulated and amplified during consequent passes by the circulating e-bunch:

$$
\frac{d P_{o p t}(\zeta)}{d n}=P_{o p t}(\zeta)\left(G(\zeta)-G_{t h}\right)+P_{S R}(\zeta)
$$

where $G_{t h}=\left(R_{1} R_{2}\right)^{-1}-1$ is loss per turn in an optical cavity with mirror reflectivity $R_{1,2}$ and $P_{S R}$ is the power of spontaneous radiation into the FEL mode. At the beginning of the process $(n=0)$ the longitudinal FEL gain profile is Gaussian, like the e-beam (2), with peak gain $G_{o}$ :

$$
G_{F E L}(\zeta)=G_{o} e^{-\zeta^{2} / 2}
$$

The complete set of dimensionless self-consistent equations for a Super-pulse can be derived for a rather general SR FEL case, using Einstein's relations between spontaneous and induced radiation $[10,11]$. The optical field in an FEL can be described as a wave packet with slowly varying complex amplitude and a normalized transverse mode function $\vec{u}(\vec{r})$ :

$$
\begin{gathered}
\overrightarrow{\mathrm{E}}_{o p t}=\operatorname{Re} \vec{u}(\vec{r}) \mathrm{A}_{o}(c t-z) e^{i k_{o}(c t-z)} ; \int|\vec{\mu}(\vec{r})|^{2} d x d y=1 ; \\
\mathrm{P}_{o p t}(c t-z)=\frac{c}{8 \pi}\left|\mathrm{A}_{o}(c t-z)\right|^{2} ;\left|\frac{d \mathrm{~A}_{o}(z)}{d z}\right|<<\frac{\left|\mathrm{A}_{o}\right|}{\Xi} ; k_{o}=\frac{2 \pi}{\lambda_{o}} .
\end{gathered}
$$

We assume that the wave-packet is not Fourier limited, which is always correct for a pulsed SR FEL $[3,5]$. Variation of electron energy in an FEL is the result of local interactions with a $\Xi$-long segment of the optical field, which modifies longitudinal motion, and can be expressed by an integral along the electron's trajectory:

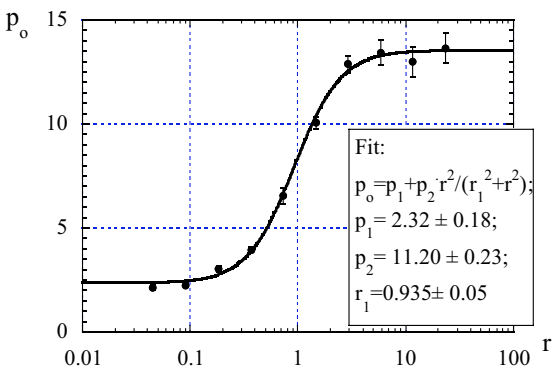

(a)

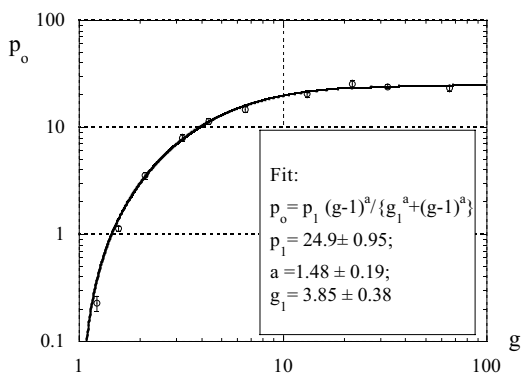

$$
\Delta E_{e}(\xi)=e \int \overrightarrow{\mathrm{E}}_{\mathrm{opt}}\left(\vec{r}_{e}, \xi\left(\vec{r}_{e}, \varepsilon\right)\right) d \vec{r}_{e} .
$$

For a short wavelength FEL with $\lambda_{o} \ll \alpha_{c} C \sigma_{\varepsilon o}$ (C is the ring circumference and $\alpha_{c}$ is the momentum compaction factor) the correlations between electrons are washed-away after one turn around the storage ring and the optical phase $\phi=k_{o}\left(c t_{i}-z_{i}\right)$ of electrons entering the FEL is random. Resultantly the connection between the average energy loss and the energy diffusion is given by [10],

$$
\langle\Delta \delta\rangle=\frac{d D_{\delta}}{d \delta} ; \quad D_{\delta}=\frac{1}{2}\left\langle\Delta \delta^{2}\right\rangle .
$$

Using the local interaction (5), local energy conservation and eq. (6), one can write a set of dimensionless equations:

$$
\begin{aligned}
& \frac{\partial f}{\partial v}+r\left(\delta \frac{\partial f}{\partial \zeta}-\zeta \frac{\partial f}{\partial \delta}\right)-p_{o}(\zeta) \cdot g \cdot \frac{\partial}{\partial \delta}\left\lceil q(\delta) \frac{\partial f}{\partial \delta}\right]=0 \\
& \frac{d p_{o}(\zeta)}{d v}=p_{o}(\zeta)\left(g \int f(\zeta, \delta) \frac{-d q(\delta)}{d \delta} d \delta-1\right)+p_{S R}(\zeta) ;
\end{aligned}
$$

where $v=n \cdot G_{t h}$ is the independent variable. The optical intra-cavity power is normalized by $P_{n}$, which is proportional to the peak power of the electron beam and the initial relative energy spread:

$$
p_{o}(\zeta)=\frac{P_{o p t}(\zeta)}{P_{n}} ; P_{n}=N_{e} m c^{2} \sigma_{\gamma o} \frac{c}{\sigma_{z o}}=\sqrt{2 \pi} \hat{P}_{e-\text { beam }} \frac{\sigma_{\gamma_{o}}}{\gamma_{o}} .
$$

Naturally, $P_{n}$ is of the same order given by the prediction from eq. (1). Equation (7) contains dimensionless parameters $g=G_{o} / G_{t h} \quad$ (gain parameter), $\quad r=\Omega_{s} / G_{t h}$ (refreshment parameter) and a dimensionless well-defined function:

$$
q(\delta)=\frac{\pi N_{e} r_{e}}{\sigma_{z o} \sigma_{\gamma o} G_{o}} \cdot \int d X^{4} f_{t}(X)\left|\int \vec{u}\left(\vec{r}_{e}\right) e^{i k_{o} \xi_{1}(X, \delta, z)} d \vec{r}_{e}\right|^{2}
$$

normalized to satisfy (4): $\int e^{-\delta^{2} / 2} \frac{d q(\delta)}{d \delta} d \delta=-1$.

As follows from eq. (7), for the optical beam to be amplified the derivative of the diffusion coefficient $q(\delta)$ should be negative at and around $\delta=0$. It means that $q(\delta)$ reaches its maximum at negative $\delta$ and minimum at positive $\delta$ (see Fig. 4).Choosing $\boldsymbol{N}_{\boldsymbol{D}}$ in the range between the maximum gain and the maximum average lasing power [6]:

$$
1 \leq 4 \pi\left(N_{w}+N_{D}\right) \sigma_{\varepsilon o} \leq \sqrt{g}
$$

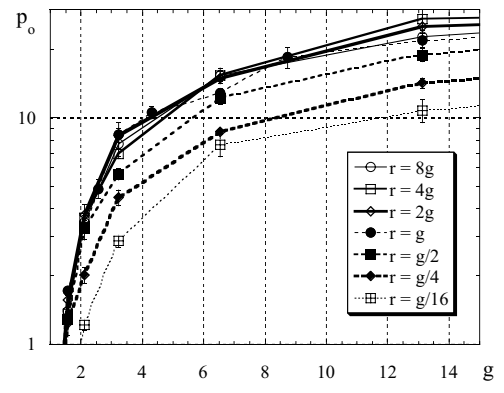

FIG.2. Dependencies of the normalized peak power $p_{o}$ : (a) on the refreshment parameter for $g=8.14$; (b)on the gain parameter $g$ for $r=10$; and (c) on the gain parameter for a number of fixed ratios $r / g=\{1 / 16,1 / 4,1 / 2,1,2,4,8\}$. Other parameters are the same as in Fig.1. Each point in the graphs shows the average value and standard error for eight to sixteen statistically independent runs of $u v f e l$ code. 
provides for attainment of maximal peak power in Super-pulse mode and $|q(\delta)| \sim 1 ; \quad|d q(\delta) / d \delta| \sim 1 .^{5}$

Our studies show very strong dependence of peak power on both the gain and refreshment parameters as shown in Fig. 2. A summary of the dependencies in Fig. 2:

(a) an FEL operating close to the threshold $(g-1<<1)$ has very low power compared with the $g>2$ case, while the increase of gain far above $g=10$ does not cause further enhancement;

(b) at $r<<1$, the synchrotron motion is essentially frozen providing for fast depletion of the local phase space density of electrons, while the increase of the refreshment factor well above ten does not increase the peak power;

(c) maximum peak power is attainable, when the ratio between $r$ and $g$ is near $\pi$, i.e. when electrons go through half of a synchrotron oscillation during one e-growth time $v_{e}=1 / g$. Thus slow synchrotron oscillations with $r<<g$ can reduce the peak power by a factor of 5-to- 6 . Nevertheless, the increase of the refreshment parameter to $r>>4 g$ does not further increase the power and can even slightly decrease it by causing oscillations in optical power.

The peak power has its strongest dependence on the gain parameter. The peak power typically increases by an order of magnitude when $g$ grows from 2 to 9 . This dependence flattens at $g>10$. Fits in Figs. 2(a) and 2(b) provide additional information, which can be used for assessing capabilities of a SR FEL in Super-pulse mode.
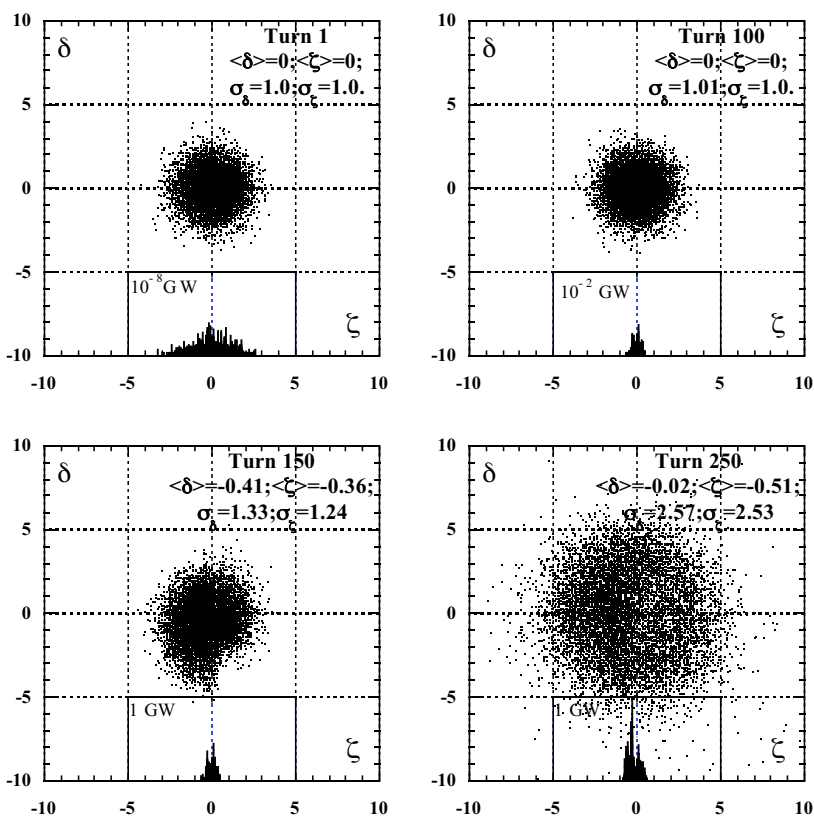

FIG.3. Four Poincaré plots in $(\delta, \zeta)$ phase-space during 250 turns in Super pulse mode. FEL parameters are the same as in Fig.2. The clips in the mid-bottoms show the $\zeta$-profiles of the optical power. The power scales are $10 \mathrm{~W}$ for turn $1,10 \mathrm{MW}$ for turn 100 and $1 \mathrm{GW}$ for turns 150 and 250 .

In an FEL with positive net gain $\left(G_{o}>G_{t h}\right)$ the period of accumulation of spontaneous radiation is followed by a period

\footnotetext{
5 The reduction of the peak power caused by finite transverse emittances of the e-beam is well described by $F_{x, y}$ factors in ref. [6].
}

of exponential growth (Fig.2), similar to that in a SelfAmplified Spontaneous Radiation (SASE) FEL [12]. This process in SR FELs is well understood and studied theoretically in [3]. The correlation length of the optical field grows with amplification $\Gamma=\int G_{F E L}(n) d n$ as $\xi_{\text {corr }} \cong \Xi \sqrt{1+\Gamma}$, while the duration of the optical pulse shrinks $\sigma_{o p t} \propto 1 / \sqrt{1+\Gamma}$.

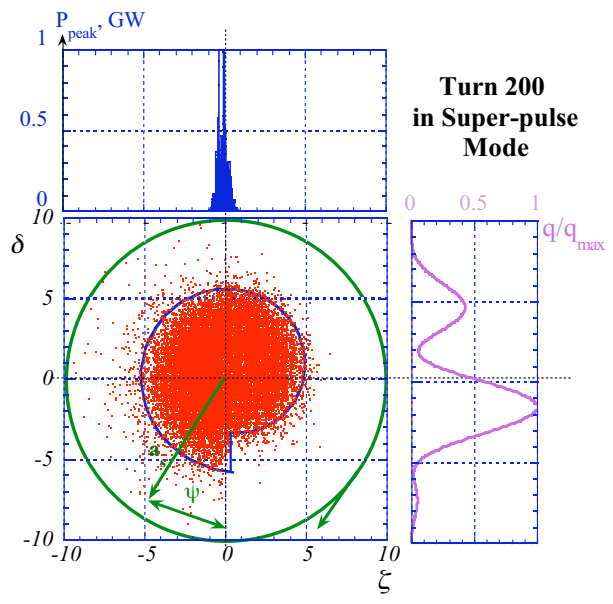

FIG.4. Poincaré plot in $(\delta, \zeta)$ phase-space for a sub-set of macroparticles at turn 200 after the start of the Super pulse. The clip on the top shows the optical power vs. $\zeta$. The clip on the right shows the energy diffusion as function of $\delta$ (see discussions below). The parameters are the same as in Fig.2. The RMS length of optical pulse is short compared with the e-bunch length: $\sigma_{\text {opt }}=0.35$. The circle and arrow shows the trajectory and direction of motion for a particle with $a=10$ (see eq. (2)) in the absence of the FEL interaction.

Fig. 3 and Fig.4 show additional details of the e-beam dynamics and provide a basis for qualitative understanding of the physics of Super-pulses. The initial exponential growth of the peak optical power by 5-to-6 orders of magnitude (Fig.3, turns 1-100) does not affect significantly the e-beam distribution or the gain profile, but the length of the optical micro-pulse reduces to about 0.3 times the initial e-bunch length. The optical pulse consists of a few tens of very short $\left(\Delta \xi=1-210^{-3}\right)$ spikes growing from the "white" noise of spontaneous radiation. The peak power in the spikes exceeds the local-average $(\Delta \xi \sim 0.1)$ value by 5 -to-20 times (see Fig.7 in ref. [4]). These spikes provide for approximately an order of magnitude increase in the peak power above the estimate given by eq. (1). The random nature of the spontaneous radiation provides for pulse-to-pulse fluctuations in peak power, which can be as large as a factor of two. The nature of the spikes and their statistics are well studied in theory of SASE FEL [12] and are out of the scope of this letter. Another order of magnitude increase in the peak power comes from the e-beam refreshment. Synchrotron oscillations continuously bring new electrons through the center region, where the optical pulse is concentrated. This allows the short optical pulse to be amplified by electrons from the e-beam periphery as well.

When the peak optical power grows to a level that affects the e-beam distribution ( 10MW for the OK-4 FEL example), the dynamics become very complicated. Energy loss and 
diffusion in the FEL make the phase-space distribution of electrons asymmetric and time dependent. The growth of the energy spread reduces the FEL gain to a level comparable to optical cavity losses while the asymmetric gain profile causes a drift of the optical pulse away from $\zeta=0$ (see Fig. 8 in ref. [4]).

The optical power reaches maximum at $G_{F E L}=G_{t h}$. After that, the processes are rather simple - the optical power decays, while electron beam distribution becomes more symmetric and simply rotates in the phase-space. This process is of interest for this letter.

Optimal Super-pulse, i.e. with maximal peak power, are generated when the growth rate of the optical power and the rate of the phase-space refreshment are well matched. Fig.4 also reveals that lower energy electrons $(-3<\delta<0)$ have maximum diffusion, while electrons with higher energy have a low diffusion energy "corridor" clearly noticeable for $1<\delta<3$. This diffusion asymmetry, which is fundamentally related to the amplification process in FEL, produces the top-bottom asymmetry in the Poincaré plots. The majority of electrons which have large amplitude synchrotron oscillations $\left(a_{s} \geq 1\right)$ interact with the intense optical pulse for a small number of turns $n_{\text {int }} \propto \sigma_{\text {opt }} / a_{s} \Omega_{s}$, while passing near the $\zeta=0$ axis.

Further on, these electrons are weakly affected by the FEL interaction and undergo nearly regular synchrotron oscillations. The diffusion process imprints the history of the FEL interaction onto the distribution of the amplitudes of synchrotron oscillation $\left(a_{s}\right)$ as a function of angle $\psi$. Specifically, the energy spread induced at angle $\psi$ records the diffusion which happened about $\Delta n \cong \psi / \Omega_{s}$ turns before the snap-shot was taken. With $Q_{s}=0.0094$ used for this example, the segment $0<\psi<\pi$ shows the diffusion imprinted at negative $\delta$ between turns 147 and 200. Similarly, the segment $\pi<\psi<2 \pi$ shows the diffusion imprinted at positive $\delta$ during the same turns. The "snail-like" dependence of the amplitude spread in Fig. 4 is a record of the evolution of the optical pulse whose peak power increased (almost linearly) from 0.35 to $1 \mathrm{GW}$ between turns 150 and 200. A linear increase of the peak power at this stage of the giant pulse is the result of the gain reduction caused by both the growth of the energy spread and depleted electron density at the location of the optical pulse. The asymmetry in diffusion is also responsible for the increase of the density at $\zeta<0$ - the synchrotron motion moves electrons with negative energy deviations towards negative $\zeta$. This asymmetry creates the dipole, sextupole and higher moments in longitudinal phase-space. The residual quadrupole oscillations (with frequency $2 \Omega_{s}$ ) can be seen in the oscillations of RMS energy spread in Fig. 1 after the end of the Super-pulse.

Attainment of high peak power requires FEL gain to be significantly higher than optical cavity losses: $G \geq(3-10) G_{t h}$, as well as reasonably high synchrotron tune. These conditions can be satisfied for most SR FELs in the visible spectral range, where highly reflective optics (loss per tern $<0.1 \%$ ) are available. In the deep-UV range (150-250 $\mathrm{nm})$ where cavity losses are at least $2 \%$ per pass, only a few SR FELs, such as
OK-4/Duke and ELETTRA with $G_{o} \sim 10-15 \%$ are capable of generating Super-pulses with GW levels of peak power.

The dimensionless nature of eq. (7) provides for the scalability of the results obtained for Super-pulses from one SR FEL to another. As follows from eq. (7), starting from the same initial distribution eq. (2), the dynamics of Super-pulses are defined by three parameters $\{r, g, q(\delta)\}$ and the spontaneous radiation. Spontaneous radiation plays the role of a seed and can be ignored at later stages of Super-pulse. Two SR FELs with the same parameters $\{r, g, q(\delta)\}$ would have the same average values and variations of the dimensionless peak power $p_{o}$. The peak Super-pulse power can be obtained through multiplication by $P_{n}$. This means that presented simulations for the OK-4/Duke SR FEL can easily be used for predicting parameters in other SR FELs.

The OK-4 FEL at Duke produced Super-pulses at wavelengths of 240-270 nm for efficient coherent harmonic generation from $130 \mathrm{~nm}$ to $37 \mathrm{~nm}$ [7]. The ELETTRA SR FEL operating typically with $6 \mathrm{~mA}$ per bunch at $1.5 \mathrm{GeV}$ [13] and optical cavity losses of $2 \%$ will generate peak intra-cavity power at the $2.5-5 \mathrm{GW}$ level, which is sufficient for generating coherent harmonics down to tens of nm. Overall, the unusual phenomena of Super pulses in SR FELs opens a range of new opportunities for SR FELs.

The author thanks Emily Longhi (Duke University) for help with preparation of this letter, Steve Hartman (Duke University) for help with the computer set-up and the Department of Physics and Dean of Natural Sciences of Duke University for financial support of this research.

[1] N.A.Vinokurov and A.N.Skrinsky, Preprints 77-59 and 77-67 (1977), BINP, Novosibirsk, Russia

[2] I.V.Pinayev, V.N.Litvinenko, S.H.Park, Y.Wu, M.Emamian, N.Hower, J.Patterson, G.Swift, Nuclear Nucl. Instr. and Meth. A 475 (2001) 222

[3] V.N.Litvinenko, N.A.Vinokurov, Nucl. Instr. and Meth. A 304 (1991) 66

[4] V.N.Litvinenko, S.H.Park, I.V.Pinayev, Y.Wu, Nucl. Instrum. and Meth. A 475 (2001) 240

[5] V.N.Litvinenko, B.Burnham, J.M.J.Madey, Y.Wu, Nucl. Instr. and Meth. A 358 (1995) 334; ibid, p. 369

[6] V.N.Litvinenko, S.H.Park, I.V.Pinayev, Y.Wu, Nucl. Instr. and Meth. A 475 (2001) 65

[7] V.N.Litvinenko, "New Results and Prospects for Harmonic Generation in Storage Ring FELs", accepted for publication in Nucl. Instr. and Meth. in Physics Research A

[8] H.Wiedemann, "Particle Accelerator Physics", Springer-Verlag, Berlin, 1993

[9] P.Elleaume, Nucl. Instr. and Meth. A 237 (1985) 28

[10] V.N.Litvinenko, N.A.Vinokurov, Nucl. Instr. and Meth. A 331 (1993) 440; S.Krinsky, J.M.Wang and P.Luchini, J.Appl. Phys. 53 (1983) 5453; J.M.J.Madey, Nouvo Cimento 50 (1979) 64

[11] A.Einstein, The set of papers in four volumes, V.3, Nauka, Moscow, 1966

[12] E.L.Saldin, E.A.Schneidmiller, M.V.Yurkov, "The Physics of Free-Electron Lasers", Springer-Verlag, Berlin, 2000; ibid. Opt. Commun. 148 (1998) 383 C.Pellegrini Nucl. Instrum. Meth. A 475 (2001) 1

[13] G. De Ninno, private communication 